\title{
TTCM-Aided SDMA-Based Two-Way Relaying
}

\author{
Wei Liang, Soon Xin Ng and Lajos Hanzo \\ School of ECS, University of Southampton, SO17 1BJ, UK. \\ Tel: +44-23-8059 3125, Fax: +44-23-8059 4508 \\ Email: \{w17g09,sxn,lh\}@ecs.soton.ac.uk, http://www-mobile.ecs.soton.ac.uk
}

\begin{abstract}
A novel power- and bandwidth-efficient Turbo Trellis Coded Modulation (TTCM) assisted Space Division Multiple Access (SDMA) based two-way relaying scheme is proposed. The scheme advocated was designed for enhancing the throughput, reliability and coverage area in a cooperative communication system. A twin-antenna Relay Node (RN) is employed for assisting a pair of users, where each user is equipped with a single-antenna mobile unit. During the first transmission period, both users transmit their TTCM-encoded signals to the RN. The twin-antenna RN then detects these signals using various SDMA-based detection algorithms. Iterative SDMA and TTCM detection is invoked at the RN, which then broadcasts the re-encoded TTCM signals to both users during the second transmission period. Finally, each user retrieves the opposite user's signals received from the RN. Our proposed scheme outperforms the non-cooperative TTCM scheme by approximately $5.3 \mathrm{dBs}$ at a BER of $10^{-6}$, when communicating over uncorrelated Rayleigh fading channels.
\end{abstract}

Index Terms-Turbo Trellis Coded Modulation (TTCM), Space Division Multiple Access (SDMA), two-way relaying, Multi-User Detector (MUD), power sharing.

\section{INTRODUCTION}

Turbo Trellis Coded Modulation (TTCM) [1] is a joint coding and modulation scheme that has a structure similar to binary turbo codes [2], [3], where two identical parallel-concatenated Trellis Coded Modulation (TCM) [4] schemes are employed as component codes. The TTCM schemes in [1] were designed based on the search for the best component TCM codes using the so-called 'punctured' minimal distance criterion for communicating over the Additive White Gaussian Noise (AWGN) channel. Recently, various TTCM schemes were designed in [5] with the aid of Extrinsic Information Transfer (EXIT) charts [6], [7] and union bounds for approaching the capacity of the Rayleigh fading channel.

Space Division Multiple Access (SDMA) is a bandwidth efficient scheme, which relies on the Multi-Input-Multi-Output (MIMO) design philosophy. Explicitly, the transmitted signal of $L$ simultaneous up-link (UL) Mobile Stations (MS) is received within the same frequency band and differentiated purely by their Channel Impulse Response (CIR). Each MS is equipped with a single transmitter antenna and their signals are received by the $P$ different receiver antennas of the Base Station (BS) [8]. Again, at the BS the individual UL signals are separated with the aid of their unique, userspecific spatial signature constituted by their CIRs, which have to be accurately estimated [8].

A TTCM-aided SDMA OFDM system was studied in [8], [9]. We have investigated a variety of SDMA-based Multi-User Detectors (MUD) [10], namely the Zero Forcing (ZF), the Minimum Mean-Square Error (MMSE), the Interference Cancellation (IC) and Maximum Likelihood (ML) MUDs. The ML MUD provides the best performance at the cost of the highest complexity. By contrast, the ZF and MMSE MUDs have a poorer performance, but impose a lower complexity. Furthermore, the TTCM-assisted IC arrangement was found to give a better performance than that of the MMSE MUD.

Relay-assisted cooperative communication schemes have been proposed in [11]. The most popular cooperative communication protocols

The financial support of the EPSRC UK and that of the European Union under the auspices of the Optimix project is gratefully acknowledged are the Decode-and-Forward (DF) and Amplify-and-Forward (AF) schemes [8], [12]. More explicitly, the attractive two-way relaying scheme of [11] assists a pair of MSs to exchange their signals with the aid of either a single or several Relay Nodes (RN) using two transmission periods. The two-way relaying protocol aims for improving the power efficiency, achievable rate and throughput.

Against this background, in this contribution, we consider a new TTCM-aided SDMA-based two-way relaying scheme constituted by a pair of users, as well as a RN. Both users transmit simultaneously to the RN during the first transmission period. Then, the RN decodes and forwards the received superposed messages to both MSs during the second transmission period. More specifically, we propose a TTCM-aided SDMA-based two-way relaying scheme, where each MS is equipped with a single UL transmit antenna, while the RN is equipped with two antennas. Two beneficial methods are employed for creating the bit sequence before TTCM-encoding at the RN. Finally, a power-sharing technique is employed for approaching the achievable throughput and for reducing the overall transmit power.

The paper is organized as follows. The system model and our novel TTCM-aided SDMA-based two-way relaying structure are described in Section II. The performance of the scheme is evaluated in Section III. Finally, our conclusions are presented in Section IV.

\section{SYSTEM MODEL}

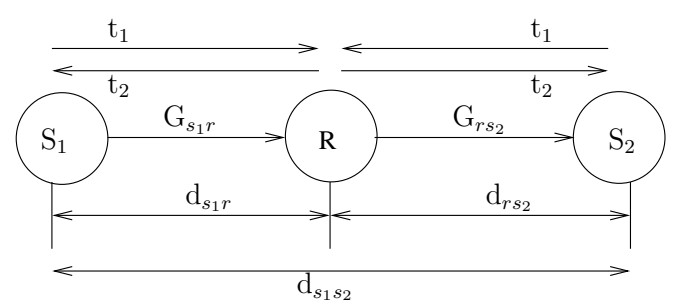

Fig. 1. Schematic of a two-way relay aided system, where $t_{1}$ is the first transmission period and $t_{2}$ is the second transmission period, $d_{a b}$ is the geographical distance between node $a$ and node $b . G_{a b}$ is the geometrical-gain between node $a$ and node $b$.

The schematic of a two-way relaying scheme is shown in Fig. 1. During the first time slot, both users transmit their information simultaneously to a RN. Then the RN decodes and forwards the received message back to the two users during the second time slot [13]-[15]. Hence, the overall system throughput is higher than that of a one-way relaying scheme, which requires two time slots to transmit one user's information.

\section{A. System Structure}

The general schematic of the TTCM-aided SDMA-based Sourceto-Relay (SR) model is shown in Fig. 2. Note that we have opted for TTCM to assist the SDMA system, since the TTCM-SDMA scheme was found to be the best arrangement from a range of coded modulation aided SDMA schemes [9].

As shown in Fig. 2, the information bit sequences $b_{1}$ and $b_{2}$ are encoded by the TTCM encoders of $\mathrm{MS}_{1}$ and $\mathrm{MS}_{2}$, respectively. The two TTCM codewords $c_{1}$ and $c_{2}$ are then fed into a virtual MIMO 


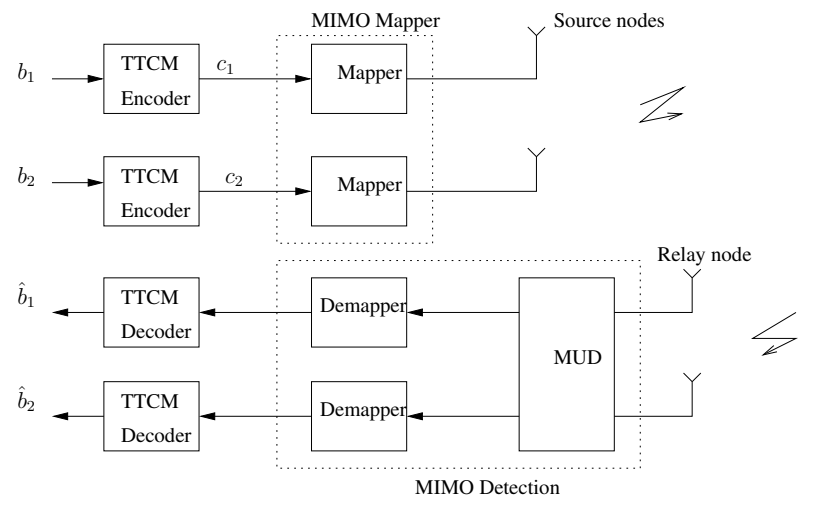

Fig. 2. The schematic of the Source Node (SN) to RN model.

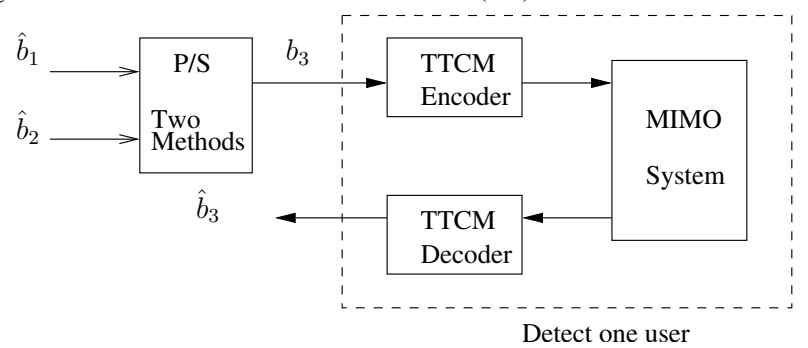

Fig. 3. The schematic of the RN to Destination Node (DN) model.The block $\mathrm{P} / \mathrm{S}$ denotes the parallel to serial converter.

mapper for transmission to the RN. Again, at the RN we consider four MUDs, namely the ML, MMSE, ZF and IC MUDs. The estimated information sequences $\hat{b}_{1}$ and $\hat{b}_{2}$ are obtained by the TTCM decoders.

As shown in Fig. 3, we consider two methods for combining the estimated information sequences $\hat{b}_{1}$ and $\hat{b}_{2}$ into $b_{3}$. The RN can concatenate the two decoded $N$-bit sequences into a $2 N$-bit sequence, i.e. we have $b_{3}=\left[\hat{b}_{1} \hat{b}_{2}\right]$. Alternatively, the RN may combine the two sequences into another $N$-bit sequence using modulo-two addition, i.e. we have $b_{3}=\hat{b}_{1} \oplus \hat{b}_{2}$, where $\oplus$ is an element-by-element modulotwo addition operator. However, the overall system throughput of the modulo-two addition aided method is higher than that of the concatenation method. The combined sequence $b_{3}$ is TTCM-encoded and broadcast from the RN to the two MSs during the second time slot. This is similar to an SDMA system using two transmit antennas and one receive antenna. Each MS then detects the signal from the opposite MS based on the TTCM-decoded sequence $\hat{b}_{3}$. For example, at the receiver of $\mathrm{MS}_{2}$, the information sequence of $\mathrm{MS}_{1}, b_{1}$, can be retrieved from the first part of $\hat{b}_{3}$, if the concatenation method is used. Alternatively, it can be retrieved from $\hat{b}_{1}=b_{2} \oplus \hat{b}_{3}$ if the modulo-two addition method is employed, where $b_{2}$ is known at the receiver of $\mathrm{MS}_{2}$.

\section{B. SDMA Channel Model}

The received signal of an SDMA system supporting $L$ users, each is equipped with a single-antenna unit, and a BS receiver equipped with $P$ antennas can be represented by [8], [9]:

$$
Y=H X+n
$$

where the received signal is a $(P \times 1)$-dimensional vector $Y=$ $\left[y_{0}, y_{1}, \cdots, y_{P-1}\right]^{T}$. Still referring to Eq. (1), the transmitted signal is an $(L \times 1)$-dimensional vector $X=\left[x_{0}, x_{1}, \cdots, x_{L-1}\right]^{T}$ and $n=\left[n_{0}, n_{1}, \cdots, n_{P-1}\right]^{T}$ is a $(P \times 1)$-dimensional Gaussian noise vector, which has a zero mean and a noise variance of $N_{0} / 2$ per dimension.

We consider a two-user SDMA scheme, where the two MSs are considered to be a two-transmitter virtual SN. We also consider a two-antenna aided RN. Note that we have incorporated the reducedpathloss-induced geometrical-gain [12], [16], [17] and the transmit power factor in the channel matrix of Eq. (1). Hence, the channel matrix between the two users and the two-antenna aided RN may be written as:

$$
H=\left[\begin{array}{ll}
\sqrt{G_{s_{1} r_{1}}} \sqrt{P_{T, s_{1}}} h_{s_{1} r_{1}} & \sqrt{G_{s_{2} r_{1}}} \sqrt{P_{T, s_{2}}} h_{s_{2} r_{1}} \\
\sqrt{G_{s_{1} r_{2}}} \sqrt{P_{T, s_{1}}} h_{s_{1} r_{2}} & \sqrt{G_{s_{2} r_{2}}} \sqrt{P_{T, s_{2}}} h_{s_{2} r_{2}}
\end{array}\right],
$$

where the subscript $r_{i}$ denotes the $i$ th receive antenna of the RN and the subscript $s_{j}$ denotes the $j$ th transmit antenna of the virtual twoantenna-aided SN, namely of the $j$ th user. Furthermore, we denote the geometrical-gain between antenna $a$ and antenna $b$ as $G_{a b}$, while $P_{T, a}$ represents the power transmitted from antenna $a$ and $h_{a b}$ represents the CIR coefficient between antenna $a$ and antenna $b$.

\section{Multi-user Detector}

The MMSE, ZF and IC MUD based SDMA schemes require at least the same number of receiver antennas as that of the transmit antennas [18]. We considered the MMSE, ZF, IC and ML MUDs in the SR link. However, only the ML MUD is used in the RD link, because there is only a single receive antenna at each DN. The weight matrix of the ZF MUD is defined as :

$$
W_{\mathrm{zf}}=H\left(H H^{H}\right)^{-1}
$$

where $H^{H}$ is the Hermitian transpose of the channel matrix. The ZF-detected signal can be expressed as [9]:

$$
\begin{aligned}
Z_{\mathrm{zf}} & =W_{\mathrm{zf}}^{H} Y \\
& =W_{\mathrm{zf}}^{H}(H X+n) \\
& =\left(H^{H} H\right)^{-1} H^{H} H X+\left(H^{H} H\right)^{-1} H^{H} n \\
& =X+\left(H^{H} H\right)^{-1} H^{H} n .
\end{aligned}
$$

By contrast, the weight matrix of the MMSE MUD is given by [9] :

$$
W_{\text {mmse }}=H\left(H H^{H}+N_{0} I_{P}\right)^{-1},
$$

where $I_{P}$ is a $(P \times P)$-element matrix having ones on its diagonal. More explicitly, the MMSE-detected signal can be written as:

$$
\begin{aligned}
Z_{\mathrm{mmse}}= & W_{\mathrm{mmse}}^{H} Y \\
= & \left(H^{H} H+N_{0} I_{P}\right)^{-1} H^{H} H X \\
& +\left(H^{H} H+N_{0} I_{P}\right)^{-1} H^{H} n .
\end{aligned}
$$

Furthermore, the ML MUD is a non-linear detector, which is optimal in terms of minimizing the symbol error probability, when all possible vectors are equally likely [19]. However, all possible $M^{L}$ combinations of the transmitted symbols have to be considered in a ML detector, where $M$ is the number of constellation points and $L$ is the number of transmit antennas. By contrast, the ZF, MMSE and IC MUDs only have to consider $M$ combinations for each. As seen from Eq. (5), the BER performance of the MMSE MUD is influenced by the interference introduced by the matrix $\left(H^{H} H+N_{0} I_{P}\right)^{-1} H^{H} H$, which is non-diagonal. We advocated a low-complexity MMSE-based IC MUD for improving the system performance by removing the offdiagonal elements in the $\left(H^{H} H+N_{0} I_{P}\right)^{-1} H^{H} H$ matrix.

It is clear from Eq. (3) that no residual interference persists after ZF MUD. However, some residual interference still contaminates the MMSE detected signal, as shown in Eq. (5). Our IC scheme is described as follows. We assume that 4PSK modulation is employed, where we have $M=4$. The soft estimate of a 4 PSK symbol was formulated as:

$$
\hat{x}=\sum_{i=1}^{J} P_{r}\left(x^{(i)}\right) x^{(i)},
$$


where $x^{(i)}$ is the $i$ th symbol in the 4PSK constellation and $P_{r}\left(x^{(i)}\right)$ is the probability of $x^{(i)}$. More specifically, from Eq. (5) the MMSEdetected signal can be written in a matrix format as:

$$
\left[\begin{array}{l}
z_{1} \\
z_{2}
\end{array}\right]=\left[\begin{array}{ll}
\sigma & \varsigma \\
\iota & \kappa
\end{array}\right] \times\left[\begin{array}{l}
x_{1} \\
x_{2}
\end{array}\right]+\left[\begin{array}{ll}
\omega & \varrho \\
\varpi & \psi
\end{array}\right] \times\left[\begin{array}{l}
n_{1} \\
n_{2}
\end{array}\right]
$$

where $\left[\begin{array}{rr}\sigma & \varsigma \\ \iota & \kappa\end{array}\right]$ is the $W_{\mathrm{mmse}}^{H} H$ term and $\left[\begin{array}{cc}\omega & \varrho \\ \varpi & \psi\end{array}\right]$ is the $W_{\mathrm{mmse}}^{H}$ term. The resultant noise variance in $z_{1}$ is given by:

$$
\begin{aligned}
\operatorname{var}\left(\omega n_{1}+\varrho n_{2}\right) & =|\omega|^{2} N_{0}+|\varrho|^{2} N_{0} \\
& =\left(|\omega|^{2}+|\varrho|^{2}\right) N_{0},
\end{aligned}
$$

where $N_{0} / 2$ is the original noise variance per dimension. We can detect the signal received from $\mathrm{MS}_{1}$ by removing the interference from $\mathrm{MS}_{2}$ based on Eq. (7), as follows:

$$
\begin{aligned}
\tilde{z}_{1} & =z_{1}-\varsigma x_{2} \\
& =\sigma x_{1}+\omega n_{1}+\varrho n_{2} .
\end{aligned}
$$

Similarly, the signal received from $\mathrm{MS}_{2}$ can be detected as:

$$
\begin{aligned}
\tilde{z}_{2} & =z_{2}-\iota x_{1} \\
& =\kappa x_{2}+\varpi n_{1}+\psi n_{2} .
\end{aligned}
$$

Then, $\tilde{z}_{1}$ and $\tilde{z}_{2}$ of Eqs. (9) and (10) can be fed into the corresponding TTCM decoder for detecting the corresponding information sequences.

\section{Optimum Power Sharing Between the $S N$ and RN}

The employment of an appropriate power sharing technique is proposed for apportioning the transmit power between the SN and $\mathrm{RN}$. This will allow us to reduce the overall transmission power required in the two-way cooperative relaying scheme. This is necessary, because the SR and the RD links require different received SNRs for achieving the same BER. The reason behind this is that the two-antenna virtual user at the $\mathrm{SN}$ and the $\mathrm{RN}$ constitutes a $(2 \times 2)$-element MIMO scheme, which requires a lower SNR, i.e. a lower transmit power than the $(2 \times 1)$-element RD link. Hence appropriately sharing the total transmit power between them allows us to reduce the overall power required. Naturally, in a practical system an appropriately designed agile SR power-control scheme is required for maintaining the optimum sharing of the transmit power between the SN and RN. We consider a free-space path-loss model. The corresponding reduced-pathloss-induced geometrical gains [12], [16], [17] between $\mathrm{MS}_{1}$ and the $\mathrm{RN}$ as well as between the RN and $\mathrm{MS}_{2}$ are given by:

$$
G_{s_{1} r}=\left(\frac{d_{s_{1} s_{2}}}{d_{s_{1} r}}\right)^{2}
$$

and

$$
G_{r_{2}}=\left(\frac{d_{s_{1} s_{2}}}{d_{r_{2}}}\right)^{2}
$$

respectively, where $d_{a b}$ denotes the geometrical distance between node $a$ and node $b$. If the RN is located at the mid-point between $\mathrm{MS}_{1}$ and $\mathrm{MS}_{2}$, then we have $G_{s_{1} r}=G_{r s_{2}}=4$.

The average received Signal to Noise power Ratio (SNR) peruser per-receive antenna ${ }^{1}$ at the receiver node $b$ with respect to the

\footnotetext{
${ }^{1}$ We introduced the terminology of per-user, per-receive antenna SNR for the sake of a fair comparison of the different scenarios considered and to emphasize the fact that these results may be applicable to other relaying scenarios.
}

transmitter node $a$ can be computed as:

$$
\begin{aligned}
\gamma_{R} & =\frac{P_{t, a} E\left\{\left|G_{a b}\right|\right\}}{N_{0}} \cdot \frac{\sum_{b_{i}=1}^{N_{b}} \sum_{a_{j}=1}^{N_{a}} E\left\{\left|h_{b_{i} a_{j}}\right|^{2}\right\} E\left\{\left|x_{a_{j}}\right|^{2}\right\}}{N_{b} N_{a}} \\
& =\frac{P_{t, a} G_{a b}}{N_{0}}
\end{aligned}
$$

where $N_{b}$ and $N_{a}$ are the number of antennas at node $b$ and node $a$, respectively. Furthermore, $x_{a_{j}}$ is the symbol transmitted from the $j$ th antenna of node $a, h_{b_{i} a_{j}}$ is the channel coefficient from antenna $a_{j}$ to antenna $b_{i}, P_{t, a}$ is the power transmitted from node $a$ and the expected values are given by $\mathrm{E}\left\{\left|h_{b_{i} a_{j}}\right|^{2}\right\}=1$ and $\mathrm{E}\left\{\left|x_{a_{j}}\right|^{2}\right\}=1$. We define the term transmit $S N R^{2}$ as the ratio of the power transmitted from node $a$ to the noise power encountered at the receiver of node $b$ as:

$$
\gamma_{T}=\frac{P_{t, a}}{N_{0}} .
$$

Hence, the relationship between $\gamma_{T}$ and $\gamma_{R}$ can be shown to be:

$$
\gamma_{R}=\gamma_{T} G_{a b}
$$

which is also given by

$$
\Upsilon_{R}=\Upsilon_{T}+10 \log _{10}\left(G_{a b}\right)[\mathrm{dB}],
$$

where $\Upsilon_{R}=10 \log _{10}\left(\gamma_{R}\right)$ and $\Upsilon_{T}=10 \log _{10}\left(\gamma_{T}\right)$. Let us denote the transmit SNR of $\mathrm{MS}_{1}, \mathrm{MS}_{2}$ and the RN as $\gamma_{T, s_{1}}, \gamma_{T, s_{2}}$ and $\gamma_{T, r}$, respectively. We jointly consider the two users as a single twotransmitter SN during the first time slot and the RN is located at the mid-point between the two users. Hence, the power transmitted from both MSs is considered to be equal, i.e. $\gamma_{T, s}=\gamma_{T, s_{1}}=\gamma_{T, s_{2}}$. The average transmit SNR of the system can be computed as:

$$
\begin{aligned}
\bar{\gamma}_{T} & =\frac{\gamma_{T, s}+\gamma_{T, r}}{2}, \\
& =\frac{10^{\frac{\Upsilon_{T, s}}{10}}+10^{\frac{\Upsilon_{T, r}}{10}}}{2},
\end{aligned}
$$

where we have $\Upsilon_{T, s}=10 \log _{10}\left(\gamma_{T, s}\right)$ and $\Upsilon_{T, r}=10 \log _{10}\left(\gamma_{T, r}\right)$. The proposed power sharing method is provided to minimize the overall transmit power, while ensuring that the $\mathrm{RN}$ and achieve a bit error ratio (BER) of approximately $5 \times 10^{-7}$ while the DN simultaneously achieves a BER of $10^{-6}$ at the lowest possible transmit SNR. More specifically, we first find the receive SNR required for the SR link, namely $\Upsilon_{R, s}=10 \log _{10}\left(\gamma_{R, s}\right)$, and that of the RD link, namely $\Upsilon_{R, r}=10 \log _{10}\left(\gamma_{R, r}\right)$, for achieving a BER of $5 \times 10^{-7}$. The difference between these receive SNRs is given by:

$$
\begin{aligned}
\Upsilon_{R, \Delta} & =\Upsilon_{R, r}-\Upsilon_{R, s}, \\
& =\left(\Upsilon_{T, r}+10 \log _{10}\left(G_{r s_{2}}\right)\right)-\left(\Upsilon_{T, s}+10 \log _{10}\left(G_{s_{1} r}\right)\right) \\
& =\Upsilon_{T, r}-\Upsilon_{T, s}[\mathrm{~dB}]
\end{aligned}
$$

where $\Upsilon_{R, \Delta}=10 \log _{10}\left(\gamma_{R, \Delta}\right)$. Then in the non-decible domain, the difference between these transmit SNRs is derivated as:

$$
\gamma_{R, \Delta}=\frac{\gamma_{T, r}}{\gamma_{T, s}} .
$$

By referring to Eq. (17) and the Eq. (20), the transmit SNR at the $\mathrm{SN}$ is given by:

$$
\gamma_{T, s}=\frac{2 \bar{\gamma}_{T}}{1+\gamma_{R, \Delta}} .
$$

Similarly, the transmit SNR at the RN can be formulated as:

$$
\gamma_{T, r}=\frac{2 \bar{\gamma}_{T} \gamma_{R, \Delta}}{1+\gamma_{R, \Delta}}
$$

${ }^{2}$ Although the concept of transmit SNR [16] is unconventional, because it relates the transmit power to the noise power at the receiver, which are at physically different locations, it is convenient for our discussions. 
Moreover, the overall system throughput $\xi_{s}$ of our two-way relaying scheme is given by:

$$
\xi_{s}=\frac{L I_{b}}{N_{1}+N_{2}}
$$

where $N_{1}$ denotes the number of symbol periods during the first time slot, $N_{2}$ is the number of modulated symbols transmitted from the $\mathrm{RN}$ during the second time slot, $L=2$ denotes the number of users, while $I_{b}$ is the number of information bits transmitted per user within a duration of $\left(N_{1}+N_{2}\right)$.

\section{Performance Evaluation}

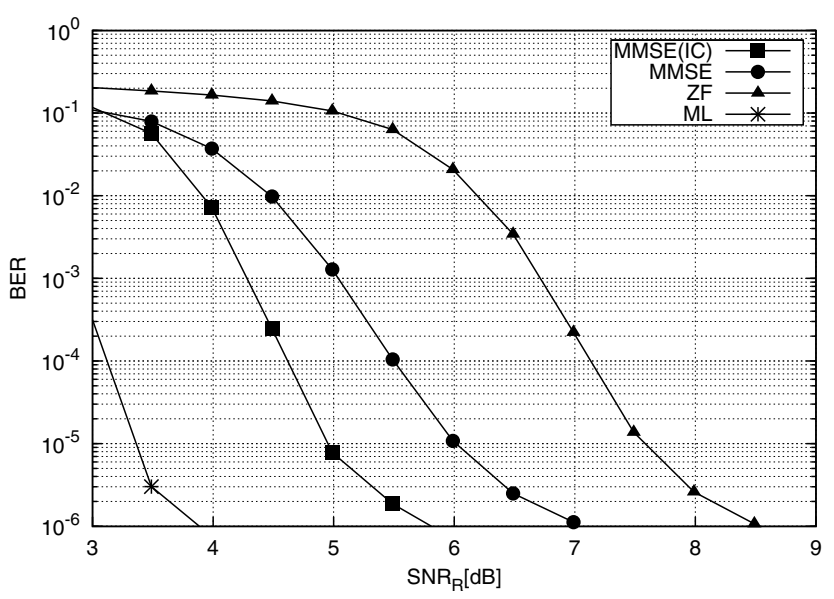

Fig. 4. BER versus received SNR per-user per-receiver antenna performance of various 4PSK-TTCM-aided SDMA schemes employing ML, MMSE, IC and ZF MUDs in the SR link. The TTCM decoder employs 4 inner iterations and 4 outer iterations for exchanging extrinsic information with the SDMA detector. The frame length is $N_{1}=1200$.

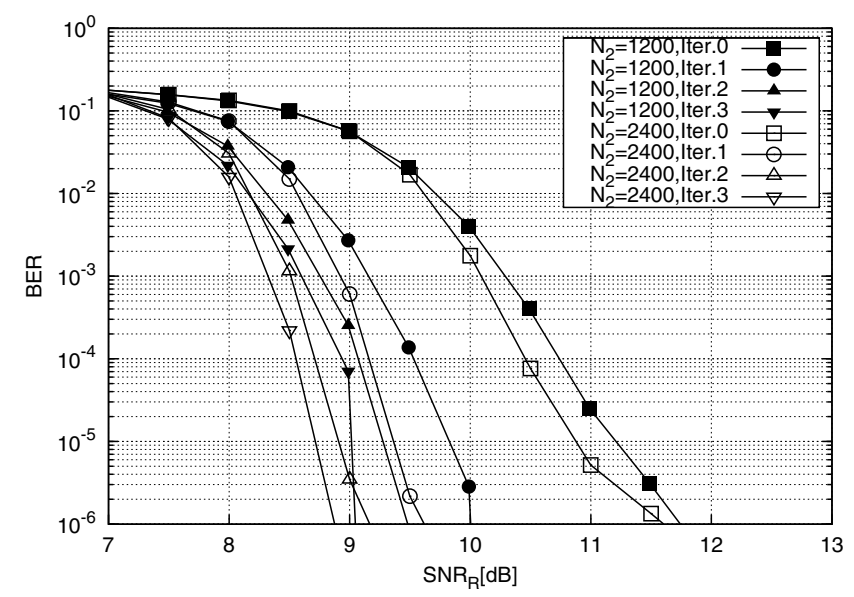

Fig. 5. BER versus received SNR per-user per-receiver antenna performance of various 4PSK-TTCM-aided SDMA schemes employing ML MUD in the RD link. The TTCM decoder employs 4 inner iterations and 4 outer iterations for exchanging extrinsic information with the SDMA detector. The frame lengths considered are $N_{2}=1200$ and $N_{2}=2400$.

An uncorrelated Rayleigh fading channel is considered and an outer iteration is defined as that when the SDMA detector and the TTCM decoder are activated once. As seen from Fig. 4, the scheme employing ML MUD that invokes four outer iterations has the best BER performance and the ZF MUD has the worst BER performance in the SR link. There is an approximately $8.5 \mathrm{~dB}-3.9 \mathrm{~dB}=4.6 \mathrm{~dB}$ difference in terms of their received SNRs at a BER of $10^{-6}$. Furthermore, after the fourth iteration the IC scheme outperforms the MMSE MUD. This is because the interfering signal introduced by the MMSE MUD is cancelled by the IC MUD. The performance of the ZF MUD cannot be further improved by having additional outer iterations, because the interfering signal has already been removed. In the first time slot, the transmitted frame length is $N_{1}=1200$ symbols.

The performance of various TTCM-aided SDMA-based schemes employing ML MUD, when communicating over the RD link during the second time slot is shown in Fig. 5. When the concatenation method of Section II-A is employed, the total number of 4PSK modulated symbols transmitted from the RN is 2400. By contrast, when the modulo-two addition method of Section II-A is employed, we have 1200 symbols. However, due to the employment of two transmit antennas at the $\mathrm{RN}$, the total transmission period is given by $N_{2}=1200$ or $N_{2}=600$ symbols, depending on whether the concatenation or the modulo-two addition method is employed, respectively. As shown in Eq. (23), the overall system throughput of the scheme employing the concatenation method is $\xi_{s}=1$ bitper-second (bps). By contrast, that of the scheme using the modulotwo addition method is given by $\xi_{s}=1.33 \mathrm{bps}$, because we have $I_{b}=1200$ information bits transmitted per user.

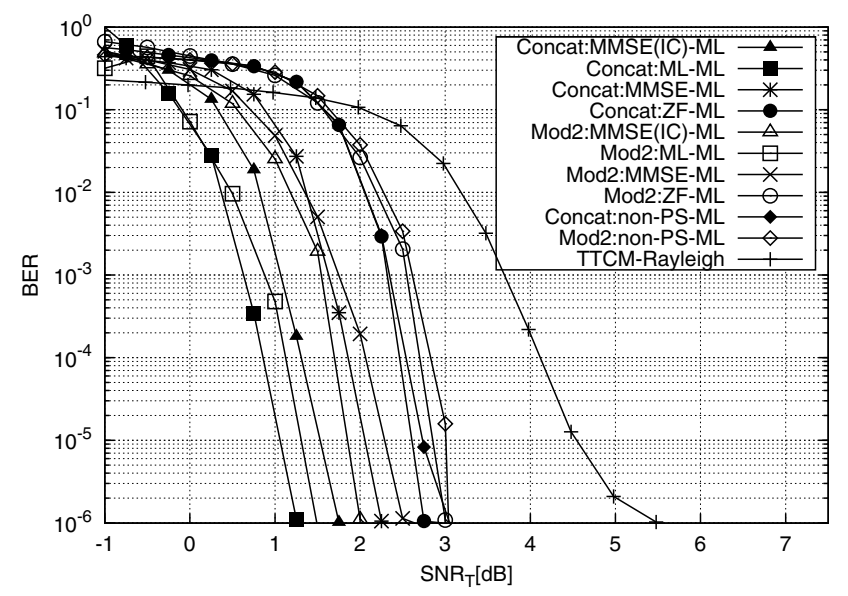

Fig. 6. BER versus transmitted SNR per user performance of various 4PSK-TTCM-aided SDMA-based two-way relaying schemes. The notation 'MMSE-ML' is used to refer to a scheme employing MMSE MUD at the SR and then the ML MUD at the RD link. Similar meaning applies to the notations 'ML-ML', 'IC-ML' and 'ZF-ML'. The modulo-two addition method is represented by ' $\bmod 2$ ' and the concatenation method is represented by 'concat'. Furthermore, all schemes employ the power sharing mechanism except those with the notation 'Non-PS'. The TTCM decoder employs 4 inner iterations and 4 outer iterations for exchanging extrinsic information with the SDMA detector. The 'TTCM-Rayleigh' scheme is our single-user benchmarker which communicates over a single transmitter and a single receiver link.

Fig. 6 portrays the BER versus transmitted SNR per user performance of various TTCM-aided SDMA-based two-way relaying schemes. We have also considered a single-user non-cooperative benchmark scheme denoted as 'TTCM-Rayleigh', where a single transmitter and a single receiver are employed. Its throughput is $1 \mathrm{bps}$. At the same throughput, the two-way relaying scheme employing the concatenation method, but operating without the power sharing mechanism, denoted as 'Concat:non-PS-ML', outperforms the 'TTCMRayleigh' benchmarker by approximately $5.5 \mathrm{~dB}-3 \mathrm{~dB}=2.5 \mathrm{dBs}$ at a BER of $10^{-6}$. When the power sharing mechanism is activated, a further $3 \mathrm{~dB}-1.2 \mathrm{~dB}=1.8 \mathrm{dBs}$ SNR gain can be attained by the 'Concate:ML-ML' scheme over the 'Concat:non-PS-ML' scheme, as seen in Fig. 6 at a BER of $10^{-6}$. The IC based scheme outperforms the MMSE and ZF based MUDs, while as expected, the ML based scheme gives the best BER performance.

Note that the SNR per bit is defined as $E_{b} / N_{0}[\mathrm{~dB}]=\mathrm{SNR}[\mathrm{dB}]-$ 


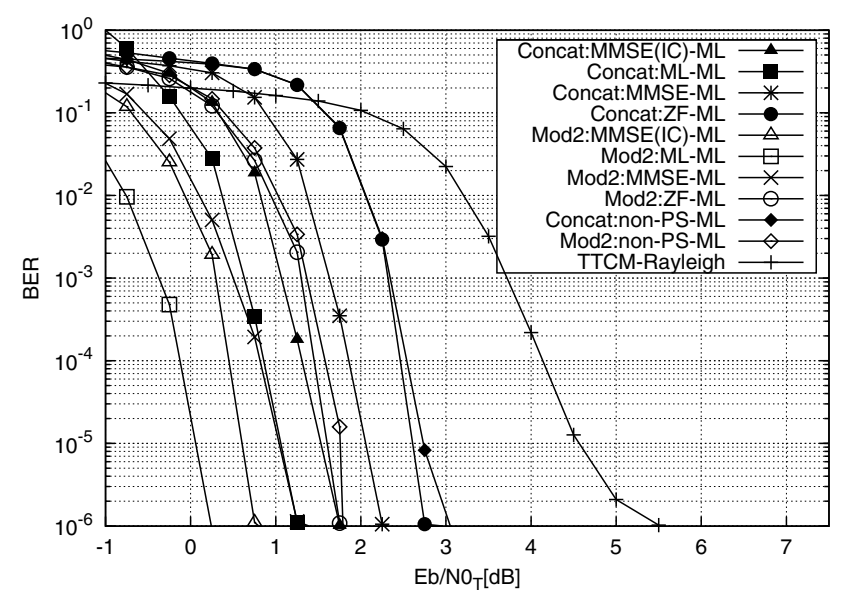

Fig. 7. BER versus transmitted $E_{b} / N_{0}$ per user performance of various 4PSK-TTCM-aided SDMA-based two-way relaying schemes. The notation 'MMSE-ML' is used to refer to a scheme employing MMSE MUD at the SR and then the ML MUD at the RD link. Similar meaning applies to the notations 'ML-ML', 'IC-ML' and 'ZF-ML'. The modulo-two addition method is represented by ' $\bmod 2$ ' and the concatenation method is represented by 'concat'. Furthermore, all schemes employ the power sharing mechanism except those with the notation 'Non-PS'. The TTCM decoder employs 4 inner iterations and 4 outer iterations for exchanging extrinsic information with the SDMA detector. The 'TTCM-Rayleigh' scheme is our single-user benchmarker which communicates over a single transmitter and a single receiver link.

$10 \log _{10}\left(\xi_{s}\right)$. Fig. 7 shows the BER versus transmit $E_{b} / N_{0}$ per user performance of various TTCM-aided SDMA-based two-way relaying schemes, which is useful for comparing the performance of the schemes employing the concatenation and the modulo-two addition methods, because they have different throughputs. The scheme employing modulo-two addition outperforms that employing the concatenation method by approximately $1 \mathrm{dBs}$ at a BER of $10^{-6}$, as seen by comparing the 'Mod2:ML-ML' and the 'Concate:MLML' curves in Fig. 7, where both schemes employ the ML MUD and the power sharing mechanism is activated. Similar improvements can be observed in Fig. 7 for the IC, MMSE and ZF based schemes, when the modulo-two addition method is employed instead of the concatenation method. As seen in Fig. 7, the 'Mod2:MLML' scheme outperforms the 'TTCM-Rayleigh' benchmark scheme by approximately $5.5 \mathrm{~dB}-0.2 \mathrm{~dB}=5.3 \mathrm{dBs}$, which is a benefit of the proposed power- and bandwidth-efficient SDMA-based twoway relaying scheme. The MMSE-detected SDMA-based two-way relay scheme offers a lower complexity at the cost of a modest $0.8 \mathrm{~dB}-0.2 \mathrm{~dB}=0.6 \mathrm{~dB}$ SNR loss in comparison to the ML-based scheme, as shown by the 'Mod2:ML-ML' and 'Mod2:IC-ML' curves in Fig. 7 at a BER of $10^{-6}$.

\section{Conclusions}

We have proposed a power- and bandwidth-efficient TTCM-aided SDMA-based two-way relaying scheme. We first quantified the achievable BER performance of the TTCM-aided SDMA schemes, when the ZF, MMSE, IC and ML MUDs are considered in the SR and RD links, respectively. Then, we invoked a power sharing mechanism to minimize the overall transmit power based on these single-link performances. The power sharing aided scheme is capable of saving approximately $1.8 \mathrm{dBs}$ of power when compared to the non-power sharing aided scheme. We have also quantified the performance of the TTCM-aided SDMA-based two-way relaying scheme, when the concatenation and modulo-two addition methods are employed at the $\mathrm{RN}$. The modulo-two addition method is capable of providing another $\mathrm{dB}$ or so SNR gain.
We found that our proposed ML-detected SDMA-based two-way relaying scheme is capable of outperforming the non-cooperative TTCM benchmark scheme by approximately $5.3 \mathrm{dBs}$ at a BER of $10^{-6}$. The MMSE detected scheme offers the best compromise in terms of the detection complexity imposed and the performance gain attained.

\section{REFERENCES}

[1] P. Robertson and T. Wörz, "Bandwidth-efficient turbo trellis-coded modulation using punctured component codes," IEEE Journal on Selected Areas in Communications, vol. 16, pp. 206-218, Feb 1998.

[2] C. Berrou and A. Glavieux and P. Thitimajshima, "Near Shannon limit error-correcting coding and decoding: Turbo codes," in Proceedings of the International Conference on Communications, (Geneva, Switzerland), pp. 1064-1070, May 1993.

[3] S. Benedetto and G. Montorsi, "Design of parallel concatenated convolutional codes," IEEE Transactions on Communications, vol. 44, pp. 591600, May 1996.

[4] G. Ungerböck, "Channel coding with multilevel/phase signals," IEEE Transactions on Information Theory, vol. IT-28, pp. 55-67, January 1982.

[5] S. X. Ng, O. Alamri, Y. Li, J. Kliewer and L. Hanzo, "Near-capacity turbo trellis coded modulation design based on EXIT charts and union bounds," IEEE Transactions on Communications, vol. 56, pp. 20302039, December 2008.

[6] S. ten Brink, "Convergence behaviour of iteratively decoded parallel concatenated codes," IEEE Transactions on Communications, vol. 49, pp. 1727-1737, October 2001.

[7] J. Kliewer, S. X. Ng, and L. Hanzo, "Efficient computation of EXIT functions for non-binary iterative decoding," IEEE Transactions on Communications, vol. 54, pp. 2133-2136, December 2006.

[8] L.Hanzo, J.Akhtman, M.Jiang, L.Wang, MIMO-OFDM for LTE, WIFI and WIMAX: coherent verus no-coherent and cooperative TurboTranceivers. John wiley-IEEE Press, 2011.

[9] M. Jiang, S. Ng, and L. Hanzo, "TCM, TTCM, BICM and BICMID assisted MMSE multi-user detected SDMA-OFDM using WalshHadamard spreading," in Vehicular Technology Conference, 2004. VTC 2004-Spring. 2004 IEEE 59th, pp. 1129 - 1133 Vol.2, May 2004.

[10] S. Verdu, Multiuser Detection, Cambridge University Press, 1998

[11] M. Chen and A. Yener, "Multiuser two-way relaying: detection and interference management strategies," IEEE Transactions on, Wireless Communications, vol. 8, no. 8, pp. $4296-4305,2009$.

[12] S. X. Ng, Y. Li, and L. Hanzo, "Distributed turbo trellis coded modulation for cooperative communications," in IEEE International Conference on Communications, 2009, pp. 1 -5, June 2009, Dresden, Germany.

[13] Y.-C. Liang and R. Zhang, "Optimal analogue relaying with multiantennas for physical layer network coding," in IEEE International Conference on Communications, 2008, pp. 3893 -3897, May 2008, Bejing, China.

[14] I. Hammerstrom, M. Kuhn, C. Esli, J. Zhao, A. Wittneben, and G. Bauch, "Mimo two-way relaying with transmit csi at the relay," in IEEE 8th Workshop on Signal Processing Advances in Wireless Communications, 2007. SPAWC 2007, pp. $1-5,2007$.

[15] T. Unger and A. Klein, "On the performance of two-way relaying with multiple-antenna relay stations," in Mobile and Wireless Communications Summit, 2007. 16th IST, pp. 1 -5, 2007, Budapest, Hungary.

[16] H, Ochiai, P. Mitran and V. Tarokh, "Design and analysis of collaborative diversity protocols for wireless sensor networks," in Proceedings of IEEE VTC Fall, (Los Angeles, USA), pp. 4645 - 4649, 26-29 September 2004.

[17] S. X. Ng, K. Zhu, and L. Hanzo, "Distributed source-coding, channelcoding and modulation for cooperative communications," in Vehicular Technology Conference Fall (VTC 2010-Fall), 2010 IEEE 72nd, pp. 1 -5 , sept. 2010.

[18] P. Wolniansky, G. Foschini, G. Golden, and R. Valenzuela, "V-blast: an architecture for realizing very high data rates over the rich-scattering wireless channel," in 1998 URSI International Symposium on Signals, Systems, and Electronics, pp. 295 -300, 1998.

[19] Iain S. Duff, A. Erisman, J. K. Reid, Direct methods for sparse matrices (Monographs on numerical analysis) Oxford: Clarendon. Oxford University Press ,1986. 